\title{
Globular Cluster Systems: Comparison with the Milky Way
}

\author{
Dante Minniti \\ Departamento de Astronomía, Pontificia Universidad Católica, Casilla \\ 306, Santiago 22, Chile
}

\begin{abstract}
The Milky Way globular cluster system serves as a reference for the study of more distant galaxies. Here I stress that there are still things we do not know about the globular cluster system of our own galaxy: their total number, their spatial motions and distances, and their IR properties. If our best reference point is not fully known, caution should be exercised when interpreting observations of distant systems. At the same time, when studying distant ellipticals it is not always advisable to rely on the Milky Way as a comparison. For example, only recently the first full calibration of the luminosity function of the globular cluster system of an elliptical galaxy was made, necessary to compare apples with apples.
\end{abstract}

\section{Introduction}

Globular clusters are old survivors, and we think that the globular cluster systems can give valuable clues about the formation of their parent galaxies. The Milky Way globular cluster system is taken as the main reference for the study of the globular cluster systems of more distant galaxies. This is because the Milky Way is without doubt the best studied galaxy, because we think that it is a typical spiral galaxy, and because we think that its globular cluster system is representative of other galaxies. It is therefore extremely important to know the globular cluster system of the Milky Way.

There has been a lot of improvement in the knowledge of the Milky Way globular cluster system, as shown by comparison of the compilation of Webbink (1985) with that of Harris (1996). We know that there are two main populations of globular clusters in the Milky Way. These are the metal-poor globulars belonging to a halo-like system (Zinn 1985, Armandroff \& Zinn 1998), and the metal-rich globulars belonging to a bulge-like system (Minniti 1995, Barbuy et al. 1998, Coté 1998). Nonetheless, there is still vast room for improvement, as these sub-systems can be better characterized. There are a few main areas where progress should be made.

\section{A Wish List for the MW Globular Cluster System}

Proper motions (and orbits) are known for only about $20 \%$ of the Milky Way globular clusters (Zoccali et al. 2001, Dinescu et al. 1999, Dauphole et al. 
1999). Unfortunately, even the nearest globular clusters were beyond the reach of Hipparcos, but the next generation of astrometric satellites (e.g. GAIA) will give us the $3-\mathrm{D}$ velocities for most globulars. Related to this, distances should be improved, especially for clusters at low galactic latitudes. This requires more searches for RR Lyr, better CMDs, and more multi-wavelength observations, in particular in the near-IR. Note that integrated IR magnitudes and colors are only available for about $30 \%$ of the Milky Way globulars. The 2MASS is supplying these much needed measurements.

It would also be important to know the total number of globular clusters in the Milky Way. I discuss this point below, since the other two topics have been extensively covered elsewhere.

\subsection{An Example: How Many GCs Are There in the MW?}

How many globular clusters formed in the Milky Way? We do not know; the initial globular cluster population could have been very large. Perhaps most of the globulars were destroyed, and we only see a few survivors. Disruption processes are enhanced in the inner regions of the Galaxy, and most missing globulars would belong to the bulge. But when a cluster is destroyed, its stars (e.g. its horizontal branch stars) survive, and would populate a similar region of the phase space. It is difficult to identify these particular stars, although we can do a simple exercise using RR Lyrae and clump giants.

Metal-poor globular clusters are rich in RR Lyrae, and the distribution of field RR Lyrae is similar to that of the globular clusters in the halo for $R>5$ kpc (e.g. Zinn 1985, Minniti 1996, Layden 1998). The density distribution of both populations follows a steep power law of the form $\rho \propto R^{-3.5}$. Recently, the microlensing experiments have discovered thousands of RR Lyrae in the inner regions of the Milky Way (e.g. Alard 1996, Alcock et al. 1997, Udalski et al. 1996), allowing to extend the density law inwards. In particular, the RR Lyrae density distribution in the MACHO bulge fields is very concentrated following the extension of a similar power law inwards. (Alcock et al. 1998, Minniti et al. 1999).

Metal-rich globular clusters have a prominent red clump. The clump giants' distribution in the bulge is very concentrated. It follows, as for the RR Lyrae, a power law with no signs of a turn-over (Minniti et al. 1999). Using these new pieces of evidence, it is fair to extrapolate the desity distribution law into the inner regions, in order to estimate the missing numbers of globulars.

Various authors have used an extrapolation in order to estimate the number of missing globulars in the inner regions. Oort (1976) pioneered the industry, arguing that $N=25$ globulars were missing in the inner 5 degrees. On the low side, Racine \& Harris (1989) predicted $N=11$ and Harris (2000) predicted $N=10$ clusters missing in the bulge. Zinn (1996) estimated that $N=30$ clusters are missing in the bulge, and Barbuy et al. (1999) argued that there were $N=15$ clusters missing in the inner 5 degrees. Taking these estimates, between 8 and $20 \%$ of the cluster population of the Milky Way is still to be discovered. This could sometimes be neglected, but more dangerous is the fact that the incompleteness is selective: inner globulars are missing, while we think that the halo population of globulars is complete. Almost all of the new globular clusters recently discovered lie in the bulge. This is illustrated by the serendipitous 
discovery of two new globulars in a very reddened field at $l, b=(10,0)$ by the 2MASS team (Hurt et al. 2000).

Let's analyze the completeness of a globular cluster survey in the bulge. The completeness $C\left(A_{V}, \rho, M_{v}, D, c\right)$ depends on field properties, such as absorption $A_{V}$ and crowding $\rho$, and intrinsic cluster properties, such as absolute magnitude $M_{v}$, distance $D$, and concentration parameter $c=\log R_{c} / R_{t}$. For 2MASS, the limiting magnitude varies by about 0.5 mag depending on crowding, but let's assume it is $K \sim 15$. Note that for a steep density law, most clusters must be located at about the same distance, and within $-10<(l, b)<10$ degrees.

In order to explore the effect of concentration and crowding, I have selected the 2MASS images of two clusters: M30 with high concentration and $M_{v}=-7.3$, $E(B-V)=0.04, D=8 \mathrm{kpc}$, and NGC2298, which is not very concentrated, with $M_{v}=-6.2, E(B-V)=0.13, D=10 \mathrm{kpc}$. We add these clusters randomly on top of crowded inner Milky Way fields at different distances from the Galactic center and from the plane, and then try to recover them by visual inspection. It is surprising to find that most times NGC2298 is not found in dense fields. This shows that even 2MASS will not be sufficient to find all globulars lost in the inner Milky Way. The cluster M30 is found more easily, though it is also lost in the most crowded fields. There are several candidates now identified in the 2MASS database (Dutra \& Bica 2001) that need to be followed up.

\section{Comparison With Extragalactic Globular Cluster Systems}

We often use the Milky Way globulars as templates to study more distant populations. It is worth stressing that by doing this we are not necessarily comparing apples with apples and pears with pears. The Milky Way is a spiral galaxy, and we should be suspicious of strong conclusions regarding the globular cluster systems of elliptical galaxies that rest on this local reference point. As a specific example, I discuss the luminosity function of globular cluster systems below.

\subsection{An Example: The Universal GC Luminosity Function}

Consider the luminosity function of the globular cluster systems, a favorite of astronomers to measure distances to elliptical galaxies. The basis for this method is the "gaussianity" of the luminosity function of the globular cluster systems of the Milky Way and M31. These are the two galaxies used as reference points, because they are close to us and our knowledge of them is fairly complete (though not better than $90 \%$, as I argued in the previous Section).

There is a tendency to talk about a "universal" globular cluster luminosity function. However, we do not know if the luminosity functions of globular cluster systems of other galaxies are bimodal, for example. Why not? This might well be, and we do not know. In fact, multimodal luminosity functions would be expected from some of the formation scenarios of globular clusters that are favored nowadays.

Enormous progress has occurred with the recent measurement of the luminosity function of the globular cluster system of the giant elliptical galaxy NGC 5128 by Rejkuba (2001). Using deep VLT observations and careful selection criteria, she reaches almost the faint end of the luminosity function of this galaxy for the first time, detecting clusters as faint as Pal 4 in NGC 5128. This is the 
deepest luminosity function of the globular cluster system of an elliptical galaxy made so far. For the first time Rejkuba (2001) is able to test the similarity of the globular cluster luminosity functions of ellipticals and spirals at the faint end. The globular cluster luminosity function of this galaxy shows a unimodal distribution, and, most importantly, supplies a valuable new calibration for the study of more distant giant ellipticals.

\section{Conclusions}

The Milky Way globular cluster system serves as a reference for the study of the globular cluster systems of more distant galaxies. There are, however, still important properties we do not know about the globular cluster system of our own galaxy. Chief among these is the total number of globular clusters of the Milky Way. We still do not know this number to $>10 \%$ accuracy. This may or may not be important for the interpretation of more distant systems: we do not know. Also, we know the spatial motions for only about $20 \%$ of the Milky Way globulars. Distances have improved rapidly in the last decade, but more IR observations are needed (only about $30 \%$ of the globular cluster population of our galaxy have IR photometry).

At the same time, there are other important properties that we do not know about the globular cluster systems of more distant galaxies. For example, we do not know if the globular cluster luminosity functions are unimodal. Previous studies do not reach deep enough to reach beyond the tip of the luminosity function. Only recently Rejkuba (2001) proved that the luminosity function of NGC5128 is unimodal using deep VLT observations. This is a great first step, though it is not proven yet that the globular cluster luminosity function is universal. Therefore, caution should be exercised when interpreting observations of distant systems.

\section{References}

Alard, C. 1996, ApJ, 458, L17

Alcock, C., et al. 1997, ApJ, 474, 217

Alcock, C., et al. 1998, ApJ, 492, 190

Armandroff, T. , \& Zinn, R. 1998, AJ, 96, 92

Barbuy, B., Bica, E., \& Ortolani, S. 1998, A\&A, 333, 117

Coté, P., 1998, A\&A, 118, 406

Dauphole, B. et al. 1996, A\&A, 313, 119

Dinescu, D. I., van Altena, W. F., Girard, T., \& Lopez, C. 1999, AJ, 117, 277

Dutra, C. M., \& Bica, E. 2001, A\&A, 376, 434

Harris, W. E. 1996, AJ, 112, 1487

Harris, W. E. 2000, "Globular Cluster Systems", in Lectures for the 1998 SaasFee Advanced School on Star Clusters (Springer: Heidelberg)

Hurt, R. L., et al. 2000, AJ, 120, 1876

Layden, A. 1998, ASP Conf. Ser. 136, 14 
Minniti, D. 1995, AJ, 109, 1663

Minniti, D. 1996, ApJ, 459, 175

Minniti, D., et al. 1999, ASP Conf. Ser. 165, 284

Oort, J. H. 1977, A\&A, 218, L97

Racine, R., \& Harris, W. E. 1989, AJ, 98, 1609

Rejkuba, M. 2001, A\&A, 369, 812

Udalski et al. 1996 Acta Astronomica, 46, 1

Webbink, R. 1985, IAU Symp. 113 on "Dynamics of Star Clusters" (Dordrecht: Reidel), 541

Zinn, R. 1985, ApJ, 293, 424

Zinn, R. 1996, ASP Conf. Ser. 92, 211

Zoccali M., et al. 2001, AJ 121, 2638

\section{Discussion}

M. Fall: Clearly, obscuration and crowding make it harder to find globular clusters near the Galactic center. But at the same time, the tidal field is stronger near the Galactic center, which means that the clusters there will have higher mean volume densities, which works in favor of their discovery.

D. Minniti: That is correct. This is fortunate, because in order to find the missing globulars we have to search in a small (though complicated) area of the sky within about 10 degrees of the Galactic center.

B. Santiago: I see a distinct hole in the MW globular cluster distribution on your last slide. Presumably you can quantify the amount of missing clusters in the centre by interpolating their density distribution inwards. Did you try this experiment?

D. Minniti: Yes, indeed there is a hole, and other authors and myself have estimated the number of missing globulars in the inner regions of the MW (see text). The conclusion is that there should be some 10-20 globulars still missing. 